\title{
Evaluations of Implanted Polypropylene Mesh After Surgical Removal Due to Eventration or Mesh Rejection
}

\author{
BO GDAN STELIAN MASTALIER MANOLESCU1', VALENTIN PO PESCU1*, MARIUS SEPTIMIU PETRUTESCU1, ANDRADA SERAFIM², \\ IZABELA CRISTINA STANCU2,3 \\ ${ }^{1}$ Colentina Clinical Hospital, Department of General Surgery, 19 - 21,Stefan cel Mare, 72202, Bucharest, Romania \\ 2University Politehnica of Bucharest, The Advanced Polymer Materials Group, 1-7 GhPolizu, 011061, Bucharest, Romania \\ ${ }^{3}$ University Politehnica of Bucharest, Faculty of Medical Engineering, 1-7 GhPolizu, 011061, Bucharest, Romania
}

\begin{abstract}
The Gold standard in parietal wall hernias is represented bymesh implantation. Both wall repair and herniaassociated complications are related to the anatomic location and biomechanics of the structures involved specificity. This work reports the results of the intraoperative and postoperative evaluation of synthetic polypropylene mesh integration. The mesh and the surrounding tissue, previously removed and preserved, have been analyzed macroscopically and at microscopic level. The tissue invaded the pores of the mesh and coated the monofilaments. The right balance between the mesh type and tissular response is hard to evaluate preoperatively, due to individual variations, but we can upgrade the criteria used to personalize the treatment for the best possible outcome.
\end{abstract}

Keywords: polypropylene, mesh integration, hernia

According to the European Hernia Society guidelines the repair of incisional hernias still has a high failure rate with long term recurrence rates above $30 \%$, even when mesh repair is performed [1].

Surgical mesh is a medical device that is used for additional support to the weakened or damaged tissue [2] Such prosthetics are currently fabricated from synthetic polymers. Aiming at the reinforcing of the abdominal wall, the use of synthetic meshes leads to improvement of patient outcomes through decreased operative time and faster recovery. Obviously, the recovery time depends on the type of hernia, the surgical approach, and, very important, the patient's condition both before and after surgery [2]. The characteristics of the meshes play a critical role in the success of such prosthetics. The list of the most important properties, as described in the literature, includes the type of material, the type of filament, tensile strength and porosity. These factors determine the weight of the mesh, its elasticity and strength and its biocompatibility [3]. The requirements for an ideal prosthesis should simultaneously respect the following: non-absorbable, have strength, flexible, non-allergenic, inert, non-carcinogenic, eventually with antibiotic properties, tissue gripping and ability to stimulate fibroblastic activity appropriate for an optimum incorporation into the host tissues. Ideally, the mesh should assist the healing process of the hernia defect by promoting the development of strong collagen fibers with formation of connective tissue in the proximity of the implant fibers. In this context, the porosity of the mesh becomes essential. The most important advantage of large pore size meshes over their traditional counterparts (with smaller pores) is represented by a strong tissue invasion through the porous mesh, also leading to the formation of a thinner and more elastic scar [5]. It is recognized that both the responsible selection of the implanted mesh and sutures as well as the meticulous surgical technique may avoid the occurrence of complications [4].

Polypropylene is one of the most popular synthetic polymers used as alloplastic material for abdominal wall defects surgery. This hydrophobic synthetic polymer is used to obtain a flexible and resistant structure that quickly integrates into the adjacenttissue structures. Monofilament polypropylene meshes with large pores are resistant to infection and are well integrated due to the in-depth invasion of the implant by fibroblasts and macrophages, as well as due to the development of fibrovascular structures [6]. However, despite reported positive tissue integration, in some situations surgical reintervention is required and therefore we decided to evaluate implanted meshes that needed such surgery. The evolution of hernia surgery was and is directly related with the on growing of new polymer structures with high biocompatibility [6]. In the last decade, the fabrication of polypropylene meshes (like all other on the market), reached a fine tuning stage, where the prosthesis design was analyzed carefully and remodeled to be more effective in strengthening the abdominal wall. The final piece that could be adjusted for a better result was the surgical technique protocol. For this we decided to evaluate all the implanted meshes that needed surgical reintervention.

\section{Experimental part \\ Study}

The aim of this study is to evaluate the polypropylene meshes behaviour and integration implanted by the Chevrel technique after we standardized the anterior wall repairing protocol, on patients with relapse or rejection. Chevrel technique was selected since this procedure is considered safe and effective, easy to perform and reliable even in cases of septic risk [ 7]. The cases investigated in this work were selected from our Clinic's experience in the Colentina Clinical Hospital, from a lot of 85 patients recruited in this study from January $1^{\text {st }} 2016$ to January $31^{\text {st }} 2017$ and operated using polypropylene meshes (with both micropores and macropores) for anterior abdominal incisional hernias. For 35 patients ( 4 men) with anterior hernia Chevrel's technique was used; from those, total or partial mesh rejection occurred in only 3 cases.

\section{Alloplastic product}

The nonabsorbable meshes used in this study have been produced by knitting of extruded undyed monofilament

* email: popescu.vali.umf@yahoo.com 
polypropylene (Trulene Mesh). The diameter of the fibers is 0.1 to $0.149 \mathrm{~mm}$, while the thickness of the mesh is 0.44-0.47 mm.

\section{Macroscopic investigation}

This study is an observational one, which investigates 12 polypropylene meshes from 12 different patients with an average age of 69 years (all implanted in our hospital) and with an average implantation interval of 38 (range 6181) months. Meshes were explanted following hernia recurrence (10 cases) or mesh rejection (2 cases). The latter occurred one by infection due to an intestinal fistula (fig.1) and the former due to a hematoma (fig. 2). For all the cases reported in this work the meshes were implanted in our clinic, by our team. The prelevation of samples was performed during the reconstruction surgery, to prevent mesh overlapping, and without any additional risks for the surgical procedure. The evaluation consisted in the macroscopic intraoperative evaluation of the tissues surrounding the mesh and observation and measurements of the explants preserved in formaldehyde $10 \%$.

\section{Microstructural investigation}

Scanning electron microscopy (SEM) has been performed to reveal morpho-structural aspects essential to complement the understanding of the mesh integration. A HITACHI S2600N Scanning electron microscope has been used. A non-implanted polypropylene mesh has been used as a control for morphological and microstructural characteristics of the implant. The explanted meshes have been preserved in glutaraldehyde 4\%. Ethanol dehydrating has been performed using ethanol gradient steps, followed by drying at $37^{\circ} \mathrm{C}$. The samples have been coated with a silver layer prior the SEM analysis.

\section{Results and discussions}

The integration and reinforcing efficiency of alloplastic products depend on a panel of material characteristics, processing parameters, biomechanical, biological, anatomo-physiological factors and on the surgical act. Altogether, these aspects decide the implant-assisted regeneration of the tissue. After the implantation of a mesh, the body reacts to the synthetic material in three distinct stages. Initially, protein absorption occurs on the surface on the polymer fibers, followed, in the next stage, by cell recruitment on the newly protein-decorated mesh. The third stage is represented by the fibroid encapsulation of the knitted mesh [6].

The macroscopic and morphological analyses were performed to describe the shape, consistency, thickness, length and type of tissues around the sample.

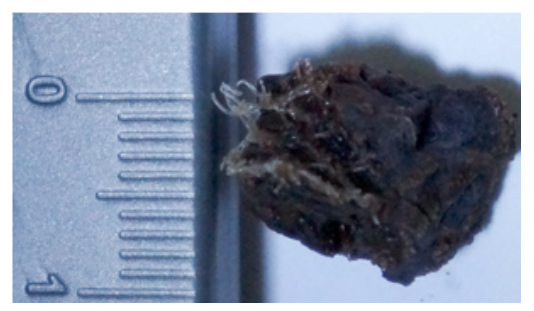

Fig.3. Close-up measurement of a fragment of blood clot that was formed under the mesh. The sample was preserved in formaldehyde $10 \%$. The structure of the mesh is still visible whitout any fibrous

deposits inspite of the 14 months implanted period.
Intraoperatively we observed that on midline prosthesis the fibroid reaction was bigger in the center (below the mesh is the sutured defect). Probably this is a result of a larger amount of growth factors released on the suturing site, which promoted the cellular migration and development, below the mesh and trough its pores, more than in the surroundings. A representative image is provided in figure 1.

In the case of the complication due to under mesh hematoma, the insufficientinteraction of the polypropylene fibers with the surrounding tissue may be noticed in figure 2 and figure 3 . In figure 3 , the large pores of the mesh are visible, with no quantitative tissue formation/ infiltration.

In seven different samples, from different patients, the mesh pattern was still distinguishable even if covered in fibroid tissue. A representative sample is imaged in figure 4 and figure 5, where an intimate contact between the mesh structure and the surrounding tissue may be noticed. The tissue has penetrated the pores of the mesh perfectly integrating the synthetic scaffold.

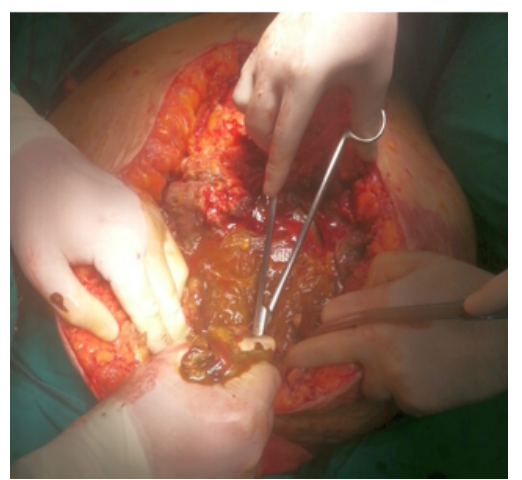

Fig 1. A midline old hernia relapse trough the polypropylene mesh with small bowel erosion due to the intimate contact between the broken mesh margin and small bowel wall. The implanted mesh pattern can be noticed at the tip of the index finger with the scissors. The mesh was explanted partially due to the tissue erosion after the intestinal bacterial contamination.
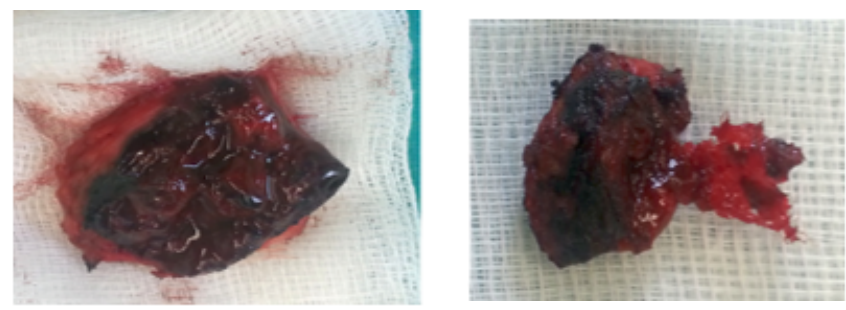

Fig. 2. Intraoperative images of the explanted polypropylene mesh after the diagnosis of under mesh hematoma: left - removed hematoma; right - a close-up of a removed clot containing the mesh
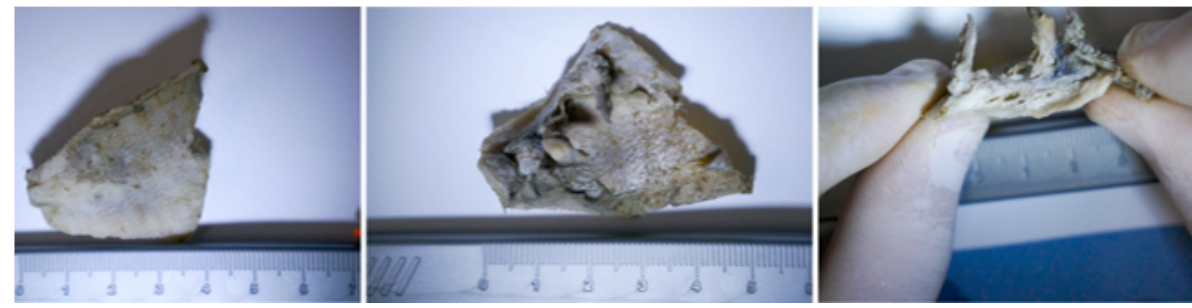

Fig.4. Measurements of the explanted polypropylene mesh after small bowel fistula. The sample was preserved in formaldehyde $10 \%$. From left to right you can see the mesh pattern on the fibroid tissue, on the middle you can see the visceral side with the fibrous and muscle tissue with the blood vessels and the last one, a section cut were you can see the layering of tissue above and below the embedded mesh (the visceral part is up) 

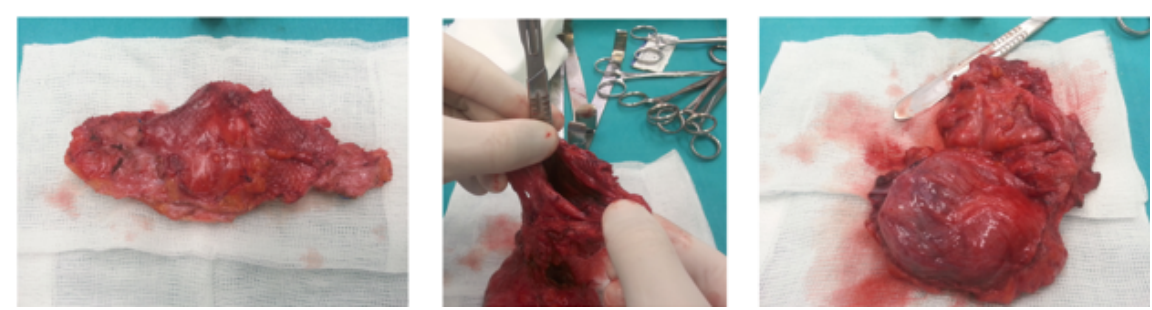

Fig. 5. Intraoperative images of the explanted polypropylene mesh for a hernia relapse. From left to right you can see the explanted tissue containing the polypropylene mesh embedded (maintaining the mesh pattern), in the middle you can see the removed hernia sac which contained small bowel segments and a piece of the great omentum; the last image is a perspective of the hernia sac removed.
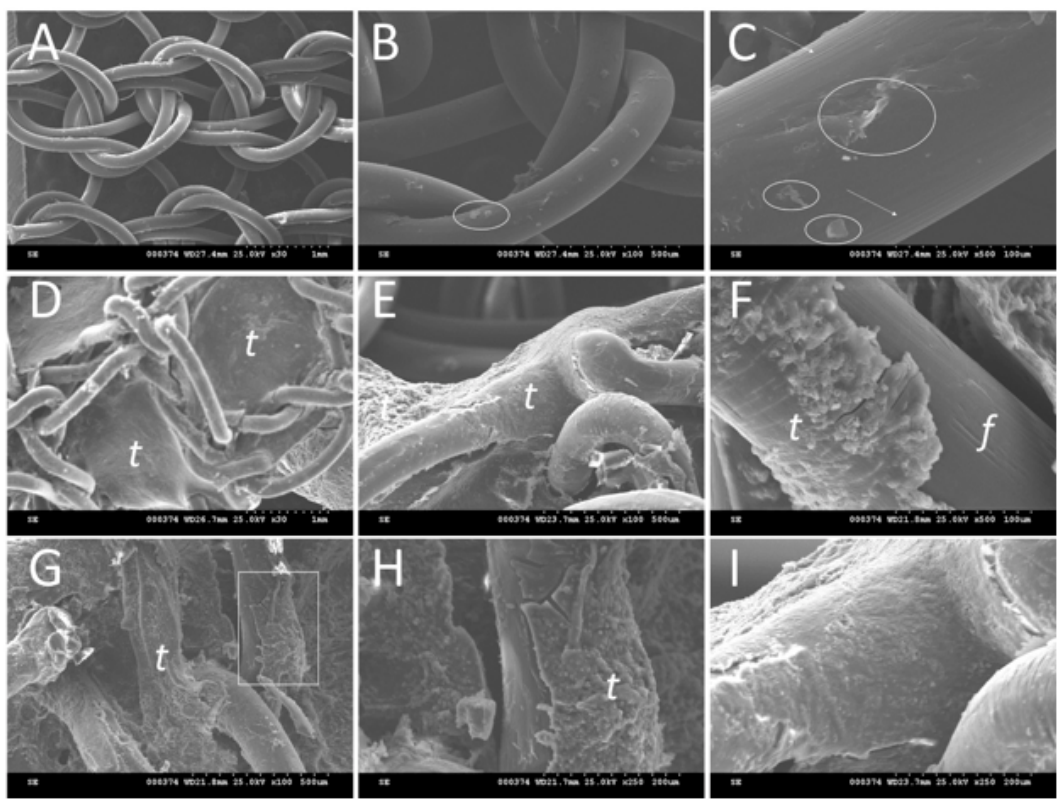

it may be noticed, for one of the explants, in figure 7D-I. Different key dimensional and morphological features were identified. The mesh pattern is more visible on the micrographs (fig. 7D) than on the explanted meshes before dehydration (e.g. left panel in figure 4 and left panel in fig. 5). This is due to the more intense contraction of the tissue following the dehydration, when compared to the knitted hydrophobic mesh whose shape is preserved. Comparing the appearance of the non-implanted control mesh (fig. $7 \mathrm{~A}$ ) with that of the explant, the size of the pores filled with tissue seems larger, most probably due to the strong invasion of the host living structures.

The tissue grew not only in the pores of the implant but also onto the surface of the polypropylene filaments, as it can be noticed in figure 7E-I. Figure 7F is representative, a monofilament is imaged with a homogeneous layer of tissue strongly adhered onto its surface. The synthetic filament may be also seen, presenting the same microstructural longitudinal superficial features characteristic to the non-implanted extruded polymer. Probably due to dehydration, a part of the covering tissue has been removed and the fibre was exposed. Strong interactions biomaterial-tissue are also revealed in figures 7G-I. This behaviour is specific to the successfully integrated meshes.

The infection of the prosthetic implanthas an incidence rate of about 1 up to $8 \%$ [8]. The most relevant factors that influence the chances of infection of the prosthesis are related to the surgical technique (mesh size, dissection 
extension, intraoperative handling), to the materials used (mesh type, sutures type) and patient-related (associated diseases, immunological status) [9].

A possible bias of the study might be related to other factors competing for the imbalance of fibroid tissue development. There are other possible factors involved in the cellular response and tissue integration of the polypropylene meshes such as tissular perfusion (poor perfusion means, less cellular activity and growth), immune status (an overrated immune response generates extended inflammation with increase chances of rejection), gender (knowing that estrogens promote wound healing [10]). Within the studied group we registered more recurrences in female patients ( 9 out of 12 patients) so the impact of estrogens could be an explanation.

It is hard to evaluate all the implants made because of poor patients' compliance, and tolerance to the relapse of the hernia. Personalising the operation for each patient's requires a good evaluation of the hernia site, dimensions, the content of hernia sac and its status, a proper porosity mesh for the local repair, managing the associated diseases for an optimal healing.In this process orienting the mesh on the proper tension lines and choosing the proper structure for the fixation plays a crucial role.

\section{Conclusions}

Polypropylene meshes perform well when used by the Chevrel's technique regarding the fibroid reaction. Postmenopausal females seem to be more affected by recurrence and poor integration, probably because of the lack of estrogens. Evaluating the morphological and structural modifications brings us more data which complete our global vision of biomaterials, especially on the in vivo behaviour of polypropylene meshes, leading to better understanding of the mesh properties and limitation and allowing further development. Knowing the behaviour of the implants can help us make a better prediction for the postoperative status, and complications development.

Acknowledgement: This work was supported by a grant of the Romanian National Authority for Scientific Research and Innovation, CNCS/CCCDI -UEFISCDI, project number PN-III-P2-2.1-PED-2016-1683, within PNCDI III.

\section{References}

1.MUYSOMS, F.E., ANTONOIOU, S.A., BURY, K., CAMPANELLI, G., CONZ,E J., CUCCURULLO, D., DE BEAUX, A.C., DEERENBERG, E.B., EAST, B.,FORTENLY, R.H., GILANES, A., JEEKEL, J., LOPEZ-CANO, M., MISEREZ, M., MORALES-CONDE, S., SANDERS, D.L., SIMONS, M.P., SEMIETAÑSKI, M., VENCLAUSKAS, L., BERREVOET, F., Hernia,19, no. 1, 2015, p.1.

2.*** FDA official website: http://www.fda.gov/MedicalDevices/ ProductsandMedicalProcedures/ImplantsandProsthetics/ HerniaSurgicalMesh/default.htm.

3.BROWN, C.N., FINCH, J.G.,Ann. R. Coll. Surg. Engl., 92, no. 4, 2010, p.272.

4.DOCTOR, H. G. J., Min. Access Surg., 2, no. 3, 2006, p.1.

5.MASTALIER, B., BOTEZATU, C.,Workshop Young Scientists J oining Forces for Excellence in Biomaterials Research, 28- 29 May 2015, Bucharest, Romania

6.PARIZA, G., MAVRODIN, C. I., ANTONIAC, V. I., Mat. Plast., 52, no. 4, 2015, p.484.

7.LICHERI, S., ERDAS, E., PISANO, G., GARAU, A., GHINAMI, E., POMATA, M. Hernia., 12, no.2, 2008, p.121.

8.FALAGAS, M.E., KASIAKOU, S.K., Clin. Microbiol. Infect., 11, no. 1, 2005, p.3.

9.MASTALIER, B., BOTEZATU, C., Metal. Int., 17, no. 8, 2012, p.143.

10.HARDMAN, M. J., ASHCROFT, G. S.,Wounds, 17, no.11, 2005, p.313.

Manuscript received: 14.12.2016 